\title{
Obstacles to strategy implementation and success factors: a review of empirical literature
}

\author{
Kristján Vigfússon \\ School of Social Sciences, Reykjavík University, Reykjavík, Iceland \\ Lára Jóhannsdóttir \\ Faculty of Business Administration, University of Iceland, Reykjavík, Iceland \\ Snjólfur Ólafsson \\ Faculty of Business Administration, University of Iceland, Reykjavík, Iceland
}

\begin{abstract}
The purpose of this study is to identify obstacles and success factors evident from empirical studies affecting strategy implementation and to provide an overview of the empirical literature. A systematic literature review (SLR) was used to analyze thirty-five peer-reviewed empirical articles. All of the 35 research papers selected in this review use primary data gathered from the organizations studied. This study of empirical research has identified 16 obstacles that can hinder strategy implementation and 18 success factors that can facilitate implementation. This study highlights factors that are dualistic, meaning that they contain both elements of obstacles and key success factors. The dualistic factors should therefore be considered in the strategy implementation process. Research gaps are identified. They include lack of studies on how to overcome obstacles to strategy on the one hand and on employee influence on implementation on the other. The differences among industries, countries, or cultures regarding implementation have not yet been explored at any depth. The paper adds to theoretical and practical knowledge based on the systematic overview it provides on strategy implementation from 1980 until 2020. The paper enriches the practical knowledge relevant to those implementing strategies, as it captures and identifies the dualistic factors affecting strategy implementation.
\end{abstract}

\section{Keywords}

strategy, implementation, execution, obstacle, barrier, success factor

\section{Introduction}

Strategy formulation has been studied and discussed in academia for half a century, but less attention has been paid to the implementation of a strategy (Aaltonen \& Ikävalko, 2002; Alexander, 1985; Al-Ghamdi, 1998; Noble, 1999a). Unlike strategy formulation, strategy implementation is extremely difficult and its research history has been seen as fragmented and eclectic (Blahová \& Knápková, 2010). Strategy implementation, nevertheless, is seen as a very important field having a crucial influence on organizations and their performance (Hrebiniak \& Joyce, 1984). Implementation is also fundamental for the effective functioning of any organization and for a business strategy to succeed (Hrebiniak, 2006; Schilit, 1987) and can be a crucial element for creating a competitive edge, which is the core aim of any strategy formulation and implementation (Giles, 1991). Multiple scholars have consequently called for more research on strategy implementation to fill the identified knowledge gap (Atkinson, 2006; Beer \& Eisenstat, 2000; Noble, 1999a). Although attention of academics has increased on strategy implementation over the last 
decade, managers are still facing remarkably similar problems concerning implementation (Hrebiniak, 2013).

Research has shown that a relatively small percentage of planned strategies in organizations are implemented (Mintzberg \& McHugh, 1985) and too many strategies seem to fail in the implementation stage because either implementation is poorly managed or has not taken place. Therefore, scholars have underlined the importance of avoiding implementation barriers and pitfalls, since most strategies falter during implementation (Allio, 2005). It is not an overstatement to say that if the strategy formulated never gets implemented, the time, resources and capital of the company have been wasted. This is, for instance, highlighted by Olson, Slater and Hult (2005. p. 47.) who state that "doing" is much harder than "dreaming." Hrebiniak (2006) agrees with Olson et al. (2005), as he emphasizes that managers know how to plan and formulate a strategy, but not how to implement it. Studies have shown a very high failure rate of strategy implementation, ranging between $50 \%$ and $90 \%$ (Kaplan \& Norton, 2001; Miller, 1997). A more recent study suggested that some of the evidence supporting these percentages is outdated, fragmentary, or lacks scientific rigor and, therefore, numbers should be taken with caution (Cândido \& Santos, 2015).

Differences between strategy implementation and strategy execution are not so clear in the literature, as many scholars use the concepts as synonyms (Hrebiniak, 2006). For instance, Li, Guo-Hui and Eppler (2010) report in their research that there seemed to be a consensus of using strategy implementation as a key word in the field of strategy implementation/execution, and that few generally use strategy execution concept (Li et al., 2010). Therefore, in this study, strategy implementation and execution will be used synonymously, in line with Hrebiniak's (2006) and Li et al. (2010) conclusion.

As depicted in Figure 1, the strategic management process can be described in three main phases: formulation, strategy implementation, and evaluation (David \& David, 2013). Inside these phases, the authors identified the following seven steps that have to be completed during the process: developing the vision and the mission for the organization, scanning the internal and external environment, setting objectives, choosing strategies, and implementing and evaluating its performance.

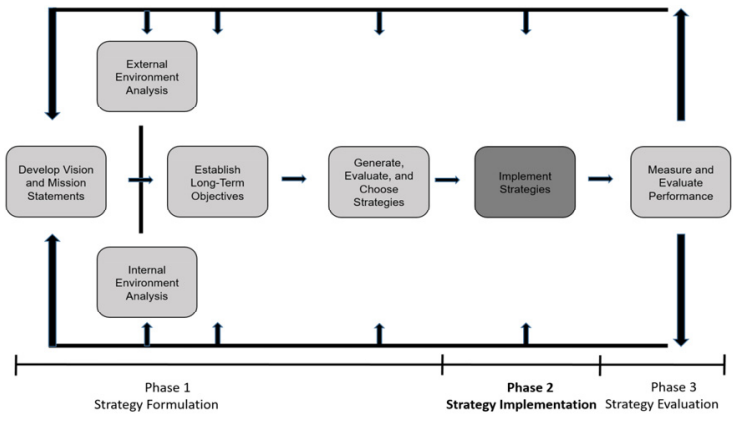

Figure 1 Model of the strategic management process (the strategy implementation phase is bolded to underline that it is the subject of this article).

Source: David \& David, 2013, p. 61

The first phase of the strategic management model (i.e., formulation) has been well studied (Miller \& Friesen, 1978; Mintzberg \& Lampel, 1999; Pettigrew, 1992) as has the role of structure and the strategy process (Galbraith \& Nathanson, 1978; Noble, 1999b). The third phase, strategy evaluation, has also been studied by a number of scholars, including Daft and Macintosh (1984), Littler, Aisthorpe, Hudson and Keasey (2000), and Simons (1994). Concerning measurement and evaluating strategy performance, which can be seen as part of the strategy evaluation method, a number of studies have also been carried out. Kaplan and Norton are among the scholars focusing on this aspect, but their emphasis has mostly been on the balanced scorecard, which is widely used to map, measure, and monitor business or corporate strategy (Kaplan \& Norton, 2000, 2004, 2008). The second phase, strategy implementation, is understudied in the literature.

A call has been made by mangers for strategy implementation guidelines (Hrebiniak, 2006). To respond to this call, scholars have introduced frameworks and conceptual models of strategy implementation (Brenes, Mena \& Molina, 2008; Hrebiniak, 2013; Okumus, 2003; Pryor, Anderson, Toombs \& Humphreys, 2007; Safdary et al., 2014). However, most of these frameworks are conceptual and descriptive and have limited practical implications (De \& Janssen, 2001; Engert \& Baumgartner, 2016).

The 5Ps model of strategy implementation, suggested by Pryor et al. (2007) is a tool that can help organizations to achieve implementation as a core competency (See Figure 2). The 5Ps model has five critical elements surrounding the concept of strategy implementation: purpose, principles, processes, people, and performance. The model also focuses on sub-elements or specific activities 
and functions, such as structures, systems, leadership behaviour, human resource policies, culture, values, and management. To ensure successful strategy implementation, the 5Ps model provides an integrated structure, which needs to be fully aligned for maximum efficiency (Pryor et al., 2007). In this study, the 5Ps model is used as an analytical framework to identify success factors and obstacles facilitating or hindering strategy implementation. The 5Ps model was chosen because not only is it conceptual but also based on integration of theory and experience from diverse business disciplines, providing an effective and pragmatic approach for strategy implementation (Pryor et al., 2007).

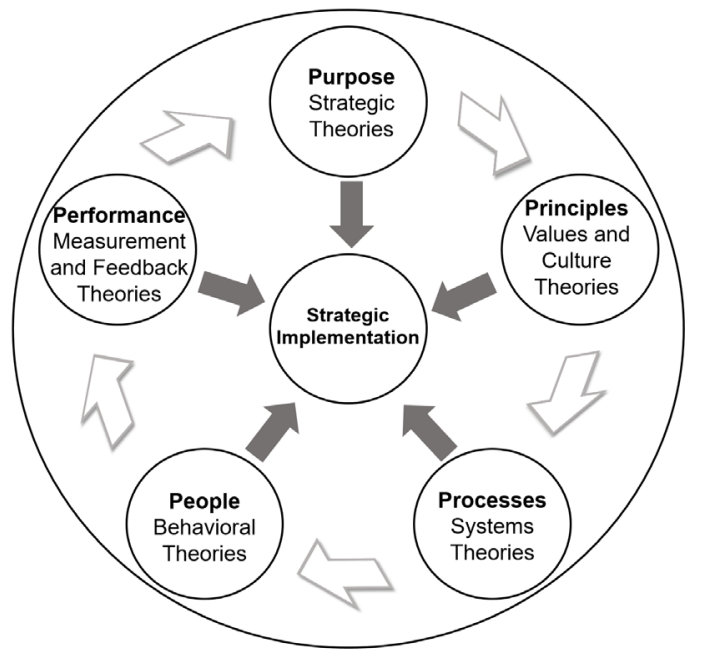

Figure $25 \mathrm{Ps}$ model of strategy implementation. Source: Pryor et al., 2007, p. 8.

At least four literature reviews on strategy implementation have been carried out, i.e. by Noble (1999a), Li et al. (2010), Alharthy, Khan, Rashid \& Pagliari (2017) and Tawse and Tabesh (2020). First, Noble (1999a) performed a mixedconceptual and empirical implementation-related review and found implementation to be a complex phenomenon and that strategy formulation and implementation are intertwined processes. Noble's findings showed three main themes of enquiry. The first theme concerned managers' behaviour, especially when managers may engage in selfinterest behaviour that can impair the implementation. The second theme covered strategic consensus among managers and key external stakeholders. The third theme related to involvement of employees as early as possible in the process, the adaptation of businesses and their culture to the changing environments, as well as managers' leadership styles (Noble, 1999a).
Second, Li et al. (2010) conducted also a mixed study of both conceptual and empirical papers, largely supporting the conclusions of Noble's (1999a). Their findings indicate that people issues received the most attention, and most of the studies explored middle managers and their influence on strategy implementation. The reviewed studies present an unclear picture of the relationship among communication, commitment, and consensus, which has been researched by a number of scholars (Alexander, 1985; Floyd \& Wooldridge, 1997; Guth \& Macmillan, 1986; Rapert, Velliquette \& Garretson, 2002). Very few studies focus on the effect that the relationships among different strategy levels have inside a company, such as business and/or operational level on implementation. In addition, there seemed to be a trend in studies towards developing frameworks and model-based approaches to strategy implementation ( $\mathrm{Li}$ et al., 2010).

Third, Alharthy et al. (2017) explored current research and a systematic search process was carried out ending with 47 papers related to the topic. As in both Li et al. and Nobles' research both conceptual and empirical papers were analysed. They found out that many organizations could not maintain competitive advantage despite having strong strategy in place. This was mostly due to lack of achievement at the strategy implementation stage as leaders and top management did not pay enough attention to the implementation. Many other factors were also neglected, and the paper specifically stated the necessity in future research to use empirical data from organizations where strategy has been implemented (Alharthy et al., 2017).

Forth, Tawse and Tabesh (2020) performed a review of the literature studying both conceptual and empirical papers focusing on organizational factors that impact strategy implementation effectiveness. The outcome of their work was an introductory framework which builds on the previous findings of Noble (1999a) identifying conditions of strategy implementation effectiveness. These conditions are the structural and interpersonal capabilities managers may employ to be able to select and carry out managerial actions that create the conditions for strategy implementation effectiveness (Tawse \& Tabesh, 2020).

The purpose of this study is to identify obstacles and success factors evident from empirical studies affecting strategy implementation. To address the issue, this study provides an overview of major 
empirical research on strategy implementation obstacles and success factors from 1980 until 2020. It identifies the major obstacles hindering strategy implementation as well as the key factors for successful implementation with a focus on identifying possible gaps in the literature. As the literature is fragmented and still in development, scholars have called for additional empirical studies to explain successful strategy implementation in different types of organizations, industries, and businesses (Baumgartner, 2014; Klettner, Clarke \& Boersma, 2014).

Empirical papers used in this literature review have been listed (See Table 1) and the empirical papers used in the four reviews done by, Noble (1999), Li et al. (2010), Alharthy et al. (2017) and Tawse and Tabesh (2020).

Table 1 Empirical papers studied in four strategy implementation reviews

\begin{tabular}{|c|c|c|c|c|c|}
\hline \multicolumn{2}{|c|}{ Papers studied in this review } & \multirow{2}{*}{\begin{tabular}{|l|} 
Papers studied \\
Noble (1999) \\
\end{tabular}} & \multirow{2}{*}{\begin{tabular}{|l|}
$\begin{array}{l}\text { Papers } \\
\text { studied }\end{array}$ \\
Li et al. (2010) \\
\end{tabular}} & \multirow{2}{*}{$\begin{array}{l}\text { Papers studied } \\
\text { Alharthy et al. (2017) }\end{array}$} & \multirow{2}{*}{\begin{tabular}{|l|} 
Papers studied \\
Tawse \& Tabesh (2020)
\end{tabular}} \\
\hline Year & Empirical research & & & & \\
\hline 1980 & Waterman, Peters \& Phillips & & & & \\
\hline 1980 & Bourgeois & $x$ & & & \\
\hline 1984 & Gupta \& Govindarajan & $x$ & $x$ & & \\
\hline 1985 & Wernham & $x$ & $x$ & & \\
\hline 1985 & Alexander & & $x$ & $\mathrm{x}$ & \\
\hline 1986 & Nutt & $x$ & $x$ & & $x$ \\
\hline 1986 & Guth \& MacMillan & $x$ & $x$ & $x$ & \\
\hline 1988 & Govindarajan & $x$ & $x$ & & $x$ \\
\hline 1989 & Hambrick \& Cannella & $x$ & & & $x$ \\
\hline 1990 & Wooldridge \& Floyd & & $x$ & & $x$ \\
\hline 1996 & Walderseel \& Sheather & $x$ & & & \\
\hline 1998 & Al - Ghamdi & & & $x$ & \\
\hline 1999 & Corboy \& O'Corrbui & & & & \\
\hline 2000 & Beer \& Eisenstat & & $x$ & $\mathrm{x}$ & $x$ \\
\hline 2001 & Rapert el al. & & $x$ & & $x$ \\
\hline 2001 & Okumus & & $x$ & & $x$ \\
\hline 2002 & Aaltonen \& Ikavalko & & & $x$ & \\
\hline 2002 & Heide, Grønhaug \& Johannessen & & $x$ & & $x$ \\
\hline 2005 & Shah & & & & \\
\hline 2006 & Hrebiniak & & $x$ & $\mathrm{x}$ & $x$ \\
\hline 2006 & Schaap & & $x$ & & $x$ \\
\hline 2008 & Crittenden \& Crittenden & & & $\mathrm{x}$ & $x$ \\
\hline 2008 & Brenes, Mena \& Molina & & $x$ & $\mathrm{x}$ & $x$ \\
\hline 2010 & Cater \& Pucko & & & & \\
\hline 2010 & $\begin{array}{l}\text { Sorooshian, Norzima, Yusof \& } \\
\text { Rosnah }\end{array}$ & & & & \\
\hline 2011 & Alamsjah & & & & \\
\hline 2012 & $\begin{array}{l}\text { Håkonsson, Burton, Obel, \& } \\
\text { Lauridsen }\end{array}$ & & & & \\
\hline 2012 & Mollahoseini \& Ahmadkhani & & & & \\
\hline 2013 & Al-Kandi, Asutay \& Dixon & & & $x$ & \\
\hline 2013 & $\begin{array}{l}\text { Pella, Sumarwan, Daryanto \& } \\
\text { Kirbrandoko }\end{array}$ & & & & \\
\hline 2015 & Sabourin & & & & \\
\hline
\end{tabular}




\begin{tabular}{l|l|l|l|l|l}
\hline 2016 & Gębczyńska & & & & \\
\hline 2018 & Köseoglu, Yazici \& Okumus & & & & \\
\hline 2019 & Tawse, Patrick \& Vera & & & & \\
\hline 2020 & Köseoglu, Altin, Chan \& Aldrag & & & & \\
\hline & Number of research & 8 & 14 & 9 & 12 \\
\hline
\end{tabular}

The structure of this article is as follows: Section 2 describes the method and analysis employed for carrying out this literature review. The results are presented and discussed in Section 3. Section 4 covers the conclusion.

\section{Research method}

The purpose of this article is to review the literature on empirical studies on strategy implementation using existing research to create new knowledge, as proposed by Torraco (2016). The research follows the guidelines offered by Nakano and Muniz (2018) on how to write a literature review for empirical papers. To fulfill the objective of building a standalone review of the empirical literature and identifying knowledge gaps concerning strategy implementation in organizations, relevant studies have been reviewed and analyzed. All of the 35 research papers selected in this review use primary data gathered from the organizations studied.

\subsection{General database search}

The method employed was to collect and analyze empirical peer-reviewed scholarly articles in the field of business strategy implementation. For this purpose, a standardized and rigorous methodology, as suggested by Nakano and Muniz (2018), was applied. It includes a useful method to build a standalone systematic literature review (SLR). The literature databases used to access the peerreviewed studies in this field were Scopus, Web of Science, and ProQuest. All searches were conducted in the search window of each database with the tools supplied and the content was displayed based on a number of criteria presented, in Table 2. The content was provided in order of relevance extracted from the titles, abstracts, and keywords of the articles.

Table 2 Criteria for systematic literature review

\begin{tabular}{l|l}
\hline Factors & Criteria for all searches \\
\hline Publication & $\begin{array}{l}\text { Peer-reviewed academic articles and book } \\
\text { chapters }\end{array}$ \\
\hline Sector & Companies and organizations \\
\hline
\end{tabular}

\begin{tabular}{l|l}
\hline Text & Full text available online \\
\hline Content & $\begin{array}{l}\text { Strategy implementation } \\
\text { obstacles/barriers/failures and levers/key } \\
\text { success factors }\end{array}$ \\
\hline $\begin{array}{l}\text { Type of } \\
\text { research }\end{array}$ & Both qualitative and quantitative studies \\
\hline Timeframe & $1980-2020$ \\
\hline Language & English \\
\hline \multicolumn{2}{c}{ Source: Nakano \& Muniz, 2018 }
\end{tabular}

\subsection{Focused searches}

The initial search results shown in Table 3 are displayed for Scopus, Web of Science, and

ProQuest databases. The numbers displayed in the Database column in Table 3 refer to the search string used for each database; the search strings are:

Search string 1; "Strategy implementation" AND "obstacle"

Search string 2; "Strategy implementation" AND "lever"

Search string 3; "Strategy implementation" AND "barrier"

Search string 4; "Strategy implementation" AND "key success factor"

Search string 5; "Strategy implementation" AND "failure"

Table 3 Results from the searched databases

\begin{tabular}{l|l|l}
\hline Scopus & $\begin{array}{l}\text { Number of articles } \\
\text { found }\end{array}$ & $\begin{array}{l}\text { Number of articles } \\
\text { relevant for this } \\
\text { research }\end{array}$ \\
\hline 1 & 26 & 5 \\
\hline 2 & 35 & 1 \\
\hline 3 & 8 & 2 \\
\hline 4 & 10 & 2 \\
\hline 5 & 46 & 1 \\
\hline Total & 125 & 11 \\
\hline ProQuest & $\begin{array}{l}\text { Number of articles } \\
\text { found }\end{array}$ & $\begin{array}{l}\text { Number of articles } \\
\text { relevant for this } \\
\text { research }\end{array}$ \\
\hline 1 & 25 & 1 \\
\hline 2 & 15 & 1 \\
\hline 3 & 115 & 0 \\
\hline 4 & 14 & 1 \\
\hline 5 & 8 & 0 \\
\hline Total & 177 & 3 \\
\hline & &
\end{tabular}




\begin{tabular}{l|l|l}
\hline $\begin{array}{l}\text { Web of } \\
\text { Science }\end{array}$ & $\begin{array}{l}\text { Number of articles } \\
\text { found }\end{array}$ & $\begin{array}{l}\text { Number of articles } \\
\text { relevant for this } \\
\text { research }\end{array}$ \\
\hline 1 & 12 & 4 \\
\hline 2 & 37 & 2 \\
\hline 3 & 9 & 0 \\
\hline 4 & 7 & 0 \\
\hline 5 & 53 & 0 \\
\hline Total & 118 & 6 \\
\hline
\end{tabular}

During the second selection, the following criteria were used for inclusion of papers:

1. Empirical studies

2. Articles published in academic journals

3. Research methods described

4. Content focused on strategy implementation obstacles and/or success factors

At this stage, 20 papers were included. This means that paper not containing evidence of strategy implementation obstacles and/or key success factors were outside the scope of this article. In other cases, the papers were outside the scope of this review if the content was not in English, even though the titles and abstracts were, or if they provided only short overviews rather than research outcomes and/or had very limited or no discussion about research methods.

\subsection{Additional searches through references}

To ensure access to articles, both open source and non-open source, the Icelandic National Library database was used, but it provides access to articles through databases such as EBSCOhost, ProQuest, Scopus, and Web of Science. In the case where access was not granted via the national database, access to articles was bought through the University of Iceland or Reykjavík University. Backward and forward snowballing technique, using the reference list and citations, was applied to identify new papers as recommended by Wohlin (2012).

An additional search of the literature was carried out in accordance with a technique recommended by Webster and Watson (2002; i.e., identifying additional references from key articles). Some of the articles referenced research and articles outside of the search scope but focused on similar topics according to their title, authors, and journals in which they were published. Therefore, 35 articles were included in the final review: 20 based on a focused search and 15 from the additional search.
The search engine Google Scholar was used to find these 15 articles.

\subsection{Analysis}

In step one, the context of the articles was analyzed based on the information available in the articles themselves. In step two, the content of the selected articles was analyzed based on the guidelines on how to write a literature review for empirical papers (Nakano \& Muniz, 2018). In step three, analytical tools were used. The analysis was conducted in three consecutive steps:

Step One: Research context

- Organizational type studied

- Strategic level

- Industry

- Countries

- Perspective

Step Two: Research objectives, findings, and methodology

- Author

- Objectives of the research

- Main findings

- Methodology

Step Three: Analysis of obstacles and success factors

- The 35 articles were categorized into two main groups: a) research focused on strategy implementation obstacles and b) research focused on strategy implementation success factors.

- The content of those research items was analysed in Excel and using the Mind Map software.

\section{Results}

As Table 4 reveals, the articles analyzed in step one deal with different contexts and external environments. The analysis was based on the type of organization studied, including private companies (Co), public organizations (PO), companies owned by either private and public owners, public/private/partnerships (PPP), and/or non-profit organizations (NPO). The studies have also been conducted on different levels within the organizations (i.e., corporate or business level or lower operational levels). Furthermore, different perspectives have been studied related to strategy implementation. Those taking part in strategy implementation can be senior and top managers, middle or lower managers, employees, or a mixture 
of all positions. Top and senior managers can be presidents, owners, board members, and/or highranking executives, such as chief executive officers
(CEO), chief finance officers (CFO), and chief operating officers (COO).

Table 4 Research context

\begin{tabular}{|c|c|c|c|c|c|}
\hline Author & $\begin{array}{l}\text { Organizatio } \\
\mathrm{n} \text { type }\end{array}$ & Strategic levels & Industries studied & $\begin{array}{l}\text { Countries } \\
\text { studied }\end{array}$ & Perspective \\
\hline Waterman (1980) & Co & $\begin{array}{l}\text { Corporate/business } \\
\text { unit }\end{array}$ & Mixed & US & Top management \\
\hline Bourgeois (1980) & $\mathrm{PO}$ & Business level & $\begin{array}{l}\text { Service, manufacturing, } \\
\text { and high-tech }\end{array}$ & US & Top management \\
\hline $\begin{array}{l}\text { Gupta \& } \\
\text { Govindarajan (1984) }\end{array}$ & Co & Business level & Mixed & US & Top management \\
\hline Wernham (1985) & $\mathrm{PO}$ & Corporate level & Telecom & UK & $\begin{array}{l}\text { Middle } \\
\text { management }\end{array}$ \\
\hline Alexander (1985) & Co & Business level & $\begin{array}{l}\text { Mixed-72 firms on the } \\
\text { Fortune } 500 \text { list- } \\
\text { industrial, retail, utilities, } \\
\text { and services }\end{array}$ & US & $\begin{array}{l}\text { Top management } \\
\text { (CEOs) }\end{array}$ \\
\hline Nutt (1986) & Co, PO, NPO & $\begin{array}{l}\text { Corporate and } \\
\text { business level }\end{array}$ & Services providers & $\begin{array}{l}\text { US \& } \\
\text { Canada }\end{array}$ & $\begin{array}{l}\text { Top management } \\
\text { (CEOs, COOs, } \\
\text { and CFOs) }\end{array}$ \\
\hline $\begin{array}{l}\text { Guth \& Macmillan } \\
\text { (1986) }\end{array}$ & Co & Business level & Mixed & US & $\begin{array}{l}\text { Middle } \\
\text { management }\end{array}$ \\
\hline Govindarajan (1988) & Co & Business level & $\begin{array}{l}\text { Automotive, petroleum, } \\
\text { food, chemical, } \\
\text { aerospace, electronics } \\
\text { retail, etc. }\end{array}$ & US & $\begin{array}{l}\text { Middle } \\
\text { management and } \\
\text { top management }\end{array}$ \\
\hline $\begin{array}{l}\text { Hambrick \& Cannella } \\
\text { (1989) }\end{array}$ & Co & Business level & Chemical & US & Top management \\
\hline $\begin{array}{l}\text { Wooldridge \& Floyd } \\
(1990)\end{array}$ & Co & Business level & $\begin{array}{l}\text { Banks and } \\
\text { manufacturers }\end{array}$ & US & $\begin{array}{l}\text { Middle } \\
\text { management }\end{array}$ \\
\hline $\begin{array}{l}\text { Walderseel \& } \\
\text { Sheather (1996) }\end{array}$ & $\mathrm{NA}$ & $\mathrm{NA}$ & $\begin{array}{l}\text { Mixed services, } \\
\text { software, insurance, etc. }\end{array}$ & Australia & $\begin{array}{l}\text { Middle managers } \\
\text { and senior } \\
\text { management }\end{array}$ \\
\hline Al-Ghamdi (1998) & $\mathrm{NA}$ & NA & $\mathrm{NA}$ & UK & NA \\
\hline $\begin{array}{l}\text { Corboy \& O'Corrbui, } \\
\text { (1999) }\end{array}$ & Co & $\mathrm{NA}$ & $\mathrm{NA}$ & US & Top management \\
\hline $\begin{array}{l}\text { Beer \& Eisenstat } \\
(2000)\end{array}$ & Co & $\begin{array}{l}\text { Corporate and } \\
\text { business level }\end{array}$ & $\mathrm{NA}$ & $\mathrm{NA}$ & $\begin{array}{l}\text { Top and middle } \\
\text { management }\end{array}$ \\
\hline Okumus (2001) & Co & $\begin{array}{l}\text { Corporate, business, } \\
\text { and operational level }\end{array}$ & Hotel services & $\mathrm{NA}$ & $\begin{array}{l}\text { Top and middle } \\
\text { management and } \\
\text { employees }\end{array}$ \\
\hline Rapert et al. (2002) & $\mathrm{NA}$ & Functional level & Health care & US & $\begin{array}{l}\text { Top and middle } \\
\text { management }\end{array}$ \\
\hline Heide et al. (2002) & Co & $\begin{array}{l}\text { Business and } \\
\text { functional levels }\end{array}$ & Ferry cruise & Norway & $\begin{array}{l}\text { Top and middle } \\
\text { management and } \\
\text { employees }\end{array}$ \\
\hline $\begin{array}{l}\text { Aaltonen \& Ikävalko } \\
\text { (2002) }\end{array}$ & $\mathrm{Co}, \mathrm{PO}$ & NA & Service industry & $\mathrm{NA}$ & $\begin{array}{l}\text { Top, middle, and } \\
\text { operational } \\
\text { management }\end{array}$ \\
\hline Shah (2005) & $\mathrm{NA}$ & NA & Mixed & India & $\begin{array}{l}\text { Middle- and } \\
\text { lower-level } \\
\text { managers } \\
\end{array}$ \\
\hline Schaap (2006) & $\mathrm{NA}$ & NA & Gaming industry & US & $\begin{array}{l}\text { Senior-level } \\
\text { managers }\end{array}$ \\
\hline Hrebiniak (2006) & $\mathrm{NA}$ & $\begin{array}{l}\text { Corporate \& business } \\
\text { level }\end{array}$ & Mixed & US & $\begin{array}{l}\text { Top and middle } \\
\text { managers }\end{array}$ \\
\hline $\begin{array}{l}\text { Crittenden \& } \\
\text { Crittenden (2008) }\end{array}$ & $\mathrm{NA}$ & NA & $\begin{array}{l}\text { Service, B2B, nonprofit } \\
\text { and consumer industries }\end{array}$ & US & NA \\
\hline
\end{tabular}




\begin{tabular}{|c|c|c|c|c|c|}
\hline Brenes et al. (2008) & $\mathrm{Co}, \mathrm{PO}$ & Business level & Mixed & $\begin{array}{l}\text { Latin } \\
\text { America }\end{array}$ & Top management \\
\hline Cater \& Pucko (2010) & Co, PO, PPP & Business level & $\begin{array}{l}\text { Mixed. Manufacturing, } \\
\text { services, trading, etc. }\end{array}$ & Slovenia & Top management \\
\hline $\begin{array}{l}\text { Sorooshian et al. } \\
(2010)\end{array}$ & NA & $\mathrm{NA}$ & $\begin{array}{l}\text { Manufacturing in } \\
\text { agriculture }\end{array}$ & Iran & Top management \\
\hline Alamsjah (2011) & Co & $\mathrm{NA}$ & $\begin{array}{l}\text { Mixed. Finance, } \\
\text { manufacturing, } \\
\text { plantation, and mining. }\end{array}$ & Indonesia & $\begin{array}{l}\text { Middle } \\
\text { management }\end{array}$ \\
\hline $\begin{array}{l}\text { Håkonsson et al. } \\
\text { (2012) }\end{array}$ & NA & $\mathrm{NA}$ & Manufacturing & $\begin{array}{l}\text { Danish } \\
\text { SMEs }\end{array}$ & Top management \\
\hline $\begin{array}{l}\text { Mollahoseini \& } \\
\text { Ahmadkhani (2012) }\end{array}$ & Co & $\mathrm{NA}$ & Food & Iran & $\begin{array}{l}\text { Executives, } \\
\text { middle } \\
\text { management, } \\
\text { operational } \\
\text { managers, and } \\
\text { experts etc. }\end{array}$ \\
\hline Al-Kandi et al. (2013) & Co & $\mathrm{NA}$ & Banking & Saudi Arabia & $\begin{array}{l}\text { Middle } \\
\text { management }\end{array}$ \\
\hline Pella et al. (2013) & NA & Business level & Mixed & Indonesia & $\begin{array}{l}\text { Top and middle } \\
\text { management }\end{array}$ \\
\hline Sabourin (2015) & $\mathrm{NA}$ & NA & Mixed & Canada & Top management \\
\hline Cebczynska (2016) & Co, PO, PPP & $\begin{array}{l}\text { Business and } \\
\text { operational levels }\end{array}$ & $\begin{array}{l}\text { Mixed. Transport, } \\
\text { finance, mining, } \\
\text { industrial process, etc. }\end{array}$ & Poland & $\begin{array}{l}\text { Top-, middle-, } \\
\text { and lower-level } \\
\text { managers }\end{array}$ \\
\hline Köseoglu et.al (2018) & NA & $\mathrm{NA}$ & Hotel business & Turkey & $\begin{array}{l}\text { Senior and } \\
\text { human resource } \\
\text { management }\end{array}$ \\
\hline Tawse et al. (2019) & $\mathrm{NA}$ & $\mathrm{NA}$ & NA & $\mathrm{NA}$ & Top management \\
\hline Köseoglu et.al (2020) & Co & Business level & Hotel business & $\begin{array}{l}\text { China/Hong } \\
\text { Kong }\end{array}$ & Top management \\
\hline
\end{tabular}

\subsection{Objectives of empirical studies, findings and methodology}

In accordance with the guidelines proposed by Nakano et al. (2018), the structure of Tables $5-8$ is based on when the relevant articles were published, author(s), research objective(s), findings, and methodology. The review included articles published between 1980 and 2020; each of the four tables covers 10 years.

In the early work of various scholars (see Table 5) related to strategy implementation, the focus was on understanding and explaining the concept of strategy in organizations in terms of how it enhances competitive advantage and improves a company's performance (Bourgeois, 1980;
Hrebiniak \& Snow, 1982; Nutt, 1983; Waterman et al., 1980; Wooldridge \& Floyd, 1990). In the 1980 s, research also focused on obstacles to strategy. Two of these early studies identified a list of obstacles that must be addressed in the formulation process, as a poor strategy formulation approach can lead to strategy obstacles later in the process (Hambrick \& Canella, 1989), whereas others need to be addressed in the implementation stage (Alexander, 1985; Wernham, 1985). As can be seen in Table 5 , studies of middle management emerged concerning how implementation factors influence middle management (Wernham, 1985) and how middle management can influence implementation (Guth \& Macmillan, 1986).

Table 5 Empirical strategy implementation literature (1980-1989)

\begin{tabular}{l|l|l|l|l}
\hline & Authors & Objective & Findings & Methodology \\
\hline 1 & $\begin{array}{l}\text { Waterman et al. } \\
(1980)\end{array}$ & $\begin{array}{l}\text { To build a model that can } \\
\text { facilitate strategy } \\
\text { implementation. }\end{array}$ & $\begin{array}{l}\text { It is not adequate to look just at strategy or } \\
\text { structure. Complex variables, like goals, } \\
\text { management styles, and skills, come into }\end{array}$ & $\begin{array}{l}\text { Case study. Interviews with } \\
\text { executives from 7 } \\
\text { companies. }\end{array}$ \\
\hline
\end{tabular}




\begin{tabular}{|c|c|c|c|c|}
\hline & & & play. & \\
\hline 2 & $\begin{array}{l}\text { Bourgeois } \\
(1980)\end{array}$ & $\begin{array}{l}\text { To identify the importance } \\
\text { of consensus, goals and } \\
\text { means. }\end{array}$ & $\begin{array}{l}\text { Strategy makers should concentrate on } \\
\text { reaching consensus rather than focusing on } \\
\text { goals when formulating a strategy. }\end{array}$ & $\begin{array}{l}\text { Questionnaires sent to top } \\
\text { management in } 12 \text { public } \\
\text { corporations. }\end{array}$ \\
\hline 3 & $\begin{array}{l}\text { Gupta \& } \\
\text { Govindarajan } \\
(1984)\end{array}$ & $\begin{array}{l}\text { To study managerial } \\
\text { characteristics affecting } \\
\text { strategy implementation. }\end{array}$ & $\begin{array}{l}\text { Unit would benefit for having managers with } \\
\text { marketing and sales experience that are } \\
\text { willing to take risks and have greater } \\
\text { tolerance to ambiguity. }\end{array}$ & $\begin{array}{l}\text { Mailed survey within } 58 \\
\text { strategic business units } \\
\text { (SBU) within eight Fortune } \\
500 \text { firms. }\end{array}$ \\
\hline 4 & $\begin{array}{l}\text { Wernham } \\
\text { (1985) }\end{array}$ & $\begin{array}{l}\text { To study how factors } \\
\text { influence the } \\
\text { implementation of strategy } \\
\text { among senior and middle } \\
\text { management. }\end{array}$ & $\begin{array}{l}\text { Factors included were the availability of } \\
\text { resources, top management support, history } \\
\text { of past implementation, and size of } \\
\text { implementation unit. }\end{array}$ & $\begin{array}{l}\text { Case study in one public } \\
\text { company (British Telecom). } \\
\text { Semi-structured interviews } \\
\text { conducted. }\end{array}$ \\
\hline 5 & $\begin{array}{l}\text { Alexander } \\
\text { (1985) }\end{array}$ & $\begin{array}{l}\text { To identify strategy } \\
\text { implementation obstacles } \\
\text { faced by top management. }\end{array}$ & $\begin{array}{l}\text { Major strategy obstacles were, lack of time } \\
\text { allocated, unexpected problems, lack of } \\
\text { coordination, and activities were not } \\
\text { effective. }\end{array}$ & $\begin{array}{l}\text { A questionnaire was } \\
\text { distributed to } 93 \text { top } \\
\text { executives. Follow-up } \\
\text { interviews with } 21 .\end{array}$ \\
\hline 6 & Nutt (1986) & $\begin{array}{l}\text { To determine if different } \\
\text { tactics affect } \\
\text { implementation. }\end{array}$ & $\begin{array}{l}\text { Four types of implementation tactics were } \\
\text { used in } 93 \% \text { of cases (intervention, } \\
\text { persuasion, participation, and edict). }\end{array}$ & $\begin{array}{l}\text { Self-report study of } 90 \\
\text { middle managers. Written } \\
\text { real-life cases. }\end{array}$ \\
\hline 7 & $\begin{array}{l}\text { Guth \& } \\
\text { Macmillan } \\
(1986)\end{array}$ & $\begin{array}{l}\text { To examine the scope and } \\
\text { effects of strategy } \\
\text { intervention. }\end{array}$ & $\begin{array}{l}\text { Middle managers who believe their self- } \\
\text { interest is compromised can redirect } \\
\text { strategy, delay, and sabotage or reduce } \\
\text { quality of implementation. }\end{array}$ & $\begin{array}{l}\text { Multi-case study with } 91 \\
\text { case studies. Data } \\
\text { collection based on in-depth } \\
\text { interviews. }\end{array}$ \\
\hline 8 & $\begin{array}{l}\text { Govindarajan } \\
\text { (1988) }\end{array}$ & $\begin{array}{l}\text { To study budget evaluative } \\
\text { style and competitive } \\
\text { strategy. }\end{array}$ & $\begin{array}{l}\text { Organization structure, control systems, } \\
\text { managers' characteristics, and firm focus are } \\
\text { effective in achieving strategy } \\
\text { implementation. }\end{array}$ & $\begin{array}{l}\text { Questionnaire used for data } \\
\text { collection from } 24 \text { firms on } \\
\text { the Fortune } 500 \text { list. }\end{array}$ \\
\hline 9 & $\begin{array}{l}\text { Hambrick \& } \\
\text { Cannella (1989) }\end{array}$ & $\begin{array}{l}\text { To study how to convert a } \\
\text { new strategy into } \\
\text { competitive success. }\end{array}$ & $\begin{array}{l}\text { Keys to implementation are, broad } \\
\text { participation at the formulation stage, } \\
\text { carefully assessing obstacles, making early } \\
\text { use of levers, selling strategy to } \\
\text { stakeholders, and responding as events } \\
\text { arise. }\end{array}$ & $\begin{array}{l}\text { A case study of one } \\
\text { company. Interviews with } \\
\text { top management. }\end{array}$ \\
\hline
\end{tabular}

From 1990 to 1999, as can be seen in Table 6, empirical research continued to centre on identifying key success factors for strategy implementation as well as strategy obstacles and pitfalls (Al-Ghamdi, 1998; Corboy \& O’Corrbui,
1999). Middle-management studies continued; one study conducted by Wooldridge and Floyd (1990) examined middle and senior managements' perception of their contributions and their strategic involvement.

Table 6 Empirical strategy implementation literature (1990-1999)

\begin{tabular}{l|l|l|l|l}
\hline & Authors & Objective & Findings & Methodology \\
\hline 10 & $\begin{array}{l}\text { Wooldridge } \\
\text { \& Floyd } \\
(1990)\end{array}$ & $\begin{array}{l}\text { To study the strategic } \\
\text { involvement of middle } \\
\text { managers. }\end{array}$ & $\begin{array}{l}\text { CEOs believe that middle managers make a } \\
\text { meaningful contribution. Middle managers } \\
\text { expect and desire strategic direction. } \\
\text { Involvement by itself does not cause } \\
\text { commitment. }\end{array}$ & $\begin{array}{l}\text { Interviews and questionnaires } \\
\text { were used to collect data from } \\
157 \text { respondents in } 20 \\
\text { organizations. }\end{array}$ \\
\hline 11 & $\begin{array}{l}\text { Walderseel } \\
\text { \& Sheather } \\
(1996)\end{array}$ & $\begin{array}{l}\text { To study if and how the } \\
\text { type of strategy } \\
\text { implemented influences } \\
\text { the implementation. }\end{array}$ & $\begin{array}{l}\text { Effects of strategy on leaders' behavior and } \\
\text { choices of implementation actions. Results } \\
\text { show that strategic context influences } \\
\text { managers' implementation intentions. }\end{array}$ & $\begin{array}{l}\text { Data were collected through } \\
\text { surveys and assignments. A } \\
\text { simulation was used to test } \\
\text { managers. Case analysis was } \\
\text { also used. }\end{array}$ \\
\hline 12 & $\begin{array}{l}\text { Al-Ghamdi } \\
(1998)\end{array}$ & $\begin{array}{l}\text { To replicate Alexanders } \\
\text { (1985) study of strategy } \\
\text { implementation. }\end{array}$ & $\begin{array}{l}\text { Six major strategy implementation obstacles } \\
\text { were identified. Communication, management } \\
\text { support, and effective information systems }\end{array}$ & $\begin{array}{l}\text { Surveys used to collect data } \\
\text { from 24 companies. }\end{array}$ \\
\hline
\end{tabular}




\begin{tabular}{l|l|l|l|l}
\hline & & & were key to successful implementation. & \\
\hline 13 & $\begin{array}{l}\text { Corboy \& } \\
\text { O'Corrbui } \\
(1999)\end{array}$ & $\begin{array}{l}\text { To identify and study } \\
\text { strategy implementation } \\
\text { obstacles. }\end{array}$ & $\begin{array}{l}\text { Individual responsibilities for implementing } \\
\text { change are not clear, senior managers } \\
\text { withdraw when implementation begins. Lack of } \\
\text { understanding of implementation. }\end{array}$ & $\begin{array}{l}\text { Role-play simulation by CEOs } \\
\text { and senior managers. }\end{array}$ \\
\hline
\end{tabular}

Between 2000 and 2009 (see Table 7), several authors presented models and frameworks based on the empirical data collected. For instance, Okumus (2001) integrated 10 key variables into a conceptual framework of implementation that was later developed into an implementation model of strategic content, context, process, and outcome (Okumus, 2003). In their conceptual framework of key dimensions of strategy implementation, Brenes et al. (2008) developed a framework based on data collected in 81 organizations in Latin America. Hrebiniak (2006) studied obstacles and how to overcome them, offering a conceptual framework on key decisions and how to translate strategy into short-term metrics and to implement it in the organizational context. Scholars continued to collect empirical data on strategy implementation obstacles and success factors throughout the decade.

Table 7 Empirical strategy implementation literature (2000-2009)

\begin{tabular}{|c|c|c|c|c|}
\hline & Authors & Objective & Findings & Methodology \\
\hline 14 & $\begin{array}{l}\text { Beer \& } \\
\text { Eisenstat } \\
(2000)\end{array}$ & $\begin{array}{l}\text { To identify strategy } \\
\text { implementation obstacles } \\
\text { and how to overcome } \\
\text { them. }\end{array}$ & $\begin{array}{l}\text { Top-down laissez-faire senior } \\
\text { management style, unclear strategy, and } \\
\text { conflicting priorities could hinder } \\
\text { successful strategy implementation. } \\
\text { Ineffective senior management team, } \\
\text { poor vertical communication, } \\
\text { coordination across functions, lack of } \\
\text { leadership skills, business, and borders } \\
\text { can affect implementation. }\end{array}$ & $\begin{array}{l}\text { A qualitative study, in total } 100 \\
\text { interviews. Meetings with top } \\
\text { managers and middle managers. A } \\
\text { total of } 150 \text { units inside } 12 \\
\text { companies were studied with an in- } \\
\text { depth examination of } 4 \text { companies. }\end{array}$ \\
\hline 15 & $\begin{array}{l}\text { Okumus } \\
\text { (2001) }\end{array}$ & $\begin{array}{l}\text { To study the } \\
\text { implementation process of } \\
\text { strategic decisions. }\end{array}$ & $\begin{array}{l}\text { Variables identified through literature } \\
\text { review play important roles in designing } \\
\text { and implementing strategies. Three new } \\
\text { variables identified: multiple project } \\
\text { implementation, organizational learning } \\
\text { and working with external companies. }\end{array}$ & $\begin{array}{l}\text { A study using interviews, } \\
\text { observations, and document } \\
\text { analysis. Case study of two hotel } \\
\text { groups. }\end{array}$ \\
\hline 16 & $\begin{array}{l}\text { Rapert et al. } \\
(2002)\end{array}$ & $\begin{array}{l}\text { To study if the frequency } \\
\text { of communication } \\
\text { between marketing and } \\
\text { top management has a } \\
\text { positive effect on strategic } \\
\text { consensus. }\end{array}$ & $\begin{array}{l}\text { Identified six strategy implementation } \\
\text { obstacles: poor vertical communication } \\
\text { and coordination across functions, top- } \\
\text { down laissez-faire senior management } \\
\text { style, unclear strategy, and conflicting } \\
\text { priorities. }\end{array}$ & $\begin{array}{l}\text { Qualitative and quantitative } \\
\text { research. A survey was mailed to } \\
1,000 \text { CEOs and marketing } \\
\text { executives with } 322 \text { responses. } \\
\text { Followed by } 30 \text { in-depth interviews. }\end{array}$ \\
\hline 17 & $\begin{array}{l}\text { Heide et al. } \\
(2002)\end{array}$ & $\begin{array}{l}\text { To identify barriers to } \\
\text { successful strategy } \\
\text { implementation. }\end{array}$ & $\begin{array}{l}\text { Different kinds of communication } \\
\text { problems were key barriers to strategy } \\
\text { implementation. }\end{array}$ & $\begin{array}{l}\text { Case study. Data were collected } \\
\text { from business unit staff members } \\
\text { through } 42 \text { structured interviews. }\end{array}$ \\
\hline 18 & $\begin{array}{l}\text { Aaltonen \& } \\
\text { lkävalko } \\
(2002)\end{array}$ & $\begin{array}{l}\text { To identify strategy } \\
\text { implementation obstacles } \\
\text { in service organizations. }\end{array}$ & $\begin{array}{l}\text { Important to align systems and structure } \\
\text { with strategy. Frequent vertical } \\
\text { communication and strategic consensus } \\
\text { can improve organizational performance. }\end{array}$ & $\begin{array}{l}\text { Qualitative research. A case study } \\
\text { of } 12 \text { service organizations with } 298 \\
\text { interviews. }\end{array}$ \\
\hline 19 & Shah (2005) & $\begin{array}{l}\text { To identify strategy } \\
\text { implementation obstacles } \\
\text { helping organizations to } \\
\text { implement strategy more } \\
\text { successfully. }\end{array}$ & $\begin{array}{l}\text { Companies most frequently experienced } \\
11 \text { obstacles during strategy } \\
\text { implementation. }\end{array}$ & $\begin{array}{l}\text { Data collected with a questionnaire } \\
\text { from } 145 \text { managers in } 35 \\
\text { industries. }\end{array}$ \\
\hline 20 & $\begin{array}{l}\text { Schaap } \\
(2006)\end{array}$ & $\begin{array}{l}\text { To study the relationship } \\
\text { between leadership } \\
\text { behavior and successful } \\
\text { strategy implementation. }\end{array}$ & $\begin{array}{l}\text { Frequent communication up and down } \\
\text { organization structure enhances } \\
\text { consensus and fosters shared values. } \\
\text { Plans must be developed and tasks, } \\
\text { timeframes, and responsibility must be } \\
\text { specified. }\end{array}$ & $\begin{array}{l}\text { Close-ended questionnaire was } \\
\text { used and mailed to the participants } \\
\text { and } 120 \text { responded. }\end{array}$ \\
\hline
\end{tabular}




\begin{tabular}{l|l|l|l|l}
\hline 21 & $\begin{array}{l}\text { Hrebiniak } \\
(2006)\end{array}$ & $\begin{array}{l}\text { To identify and evaluate } \\
\text { factors that play a } \\
\text { significant role in strategy } \\
\text { implementation. }\end{array}$ & $\begin{array}{l}\text { A strategy implementation framework } \\
\text { that consists of internal and external } \\
\text { contexts, and processes and outcomes } \\
\text { proposed. }\end{array}$ & $\begin{array}{l}\text { Survey of fixed and open-ended } \\
\text { questions. Panel discussion used. } \\
\text { Data were collected from 433 } \\
\text { managers. }\end{array}$ \\
\hline 22 & $\begin{array}{l}\text { Crittenden \& } \\
\text { Crittenden } \\
(2008)\end{array}$ & $\begin{array}{l}\text { To determine why } \\
\text { strategic planning efforts } \\
\text { and implementation fail. }\end{array}$ & $\begin{array}{l}\text { Successful implementation is based on } \\
\text { various strategic elements, such as } \\
\text { supportive policies and systems. Most } \\
\text { systematic strategy implementation } \\
\text { needs more integrative methodology. }\end{array}$ & $\begin{array}{l}\text { A mixture of primary and secondary } \\
\text { data were collected from 29 } \\
\text { organizations. }\end{array}$ \\
\hline 23 & $\begin{array}{l}\text { Brenes et al. } \\
(2008)\end{array}$ & $\begin{array}{l}\text { To measure the effect of } \\
\text { key variables on } \\
\text { successful } \\
\text { implementation. }\end{array}$ & $\begin{array}{l}\text { Five key success factors were identified } \\
\text { for implementing a business strategy } \\
\text { successfully. }\end{array}$ & $\begin{array}{l}\text { Survey questionnaire were mailed } \\
\text { and 81 companies responded. }\end{array}$ \\
\hline
\end{tabular}

In Table 8, strategy implementation and how it affects a company's performance can be seen as the main focus of studies between 2010 and 2020. For instance, Sorooshian et al. (2010) examined the relationship between strategy implementation and the performance of small- and medium-sized manufacturing companies and found that leadership style, structure, and resources supporting implementation affect financial organizational performance. Another study found that inadequate leadership and employees' reluctance to share their knowledge have a negative effect on organizational performance (Cater \&
Pucko, 2010). Furthermore, other issues affecting the organizations' performance indicators concerning implementation include not carrying out comprehensive analysis and planning and a lack of training in combination with low levels of employee motivation (Köseoglu et al., 2018). Authors continued to bridge the knowledge gap by studying factors affecting poor implementation (Mollahoseini \& Ahmadkhani, 2012; Pella et al., 2013), success factors, and the importance of organizational culture and how it can contribute to successful strategy implementation (Alamsjah, 2011; Al-Kandi et al., 2013).

Table 8 Empirical strategy implementation literature (2010-2020)

\begin{tabular}{|c|c|c|c|c|}
\hline & Authors & Objective & Findings & Methodology \\
\hline 24 & $\begin{array}{l}\text { Cater \& Pucko } \\
(2010)\end{array}$ & $\begin{array}{l}\text { To identify obstacles of } \\
\text { strategy } \\
\text { implementation and } \\
\text { how they affect } \\
\text { performance. }\end{array}$ & $\begin{array}{l}\text { The biggest obstacles having negative } \\
\text { effect on performance are inadequate } \\
\text { leadership and employees' reluctance to } \\
\text { share their knowledge. }\end{array}$ & $\begin{array}{l}\text { Questionnaires sent to } \\
\text { managers and board } \\
\text { members in } 172 \text { companies. }\end{array}$ \\
\hline 25 & $\begin{array}{l}\text { Sorooshian et al. } \\
(2010)\end{array}$ & $\begin{array}{l}\text { To study the } \\
\text { relationship between } \\
\text { strategy } \\
\text { implementation and } \\
\text { performance. }\end{array}$ & $\begin{array}{l}\text { Strategy implementation drivers, such as } \\
\text { leadership, structure, and human resource } \\
\text { management, contribute to a company's } \\
\text { performance. }\end{array}$ & $\begin{array}{l}\text { Questionnaires sent to } \\
\text { managers; } 163 \text { answered the } \\
\text { survey. }\end{array}$ \\
\hline 26 & Alamsjah (2011) & $\begin{array}{l}\text { To study interrelated } \\
\text { key success factors for } \\
\text { strategy } \\
\text { implementation by } \\
\text { middle managers. }\end{array}$ & $\begin{array}{l}\text { Five interrelated factors contribute to } \\
\text { successful implementation, including } \\
\text { clarity of strategy, corporate culture and } \\
\text { effective management communication. }\end{array}$ & $\begin{array}{l}\text { Survey of fixed questions and } \\
\text { open-ended questions aimed } \\
\text { at } 158 \text { middle-level } \\
\text { managers. }\end{array}$ \\
\hline 27 & $\begin{array}{l}\text { Håkonsson et al. } \\
\text { (2012) }\end{array}$ & $\begin{array}{l}\text { To study how and if an } \\
\text { executive style } \\
\text { determines strategy } \\
\text { implementation and } \\
\text { performance. }\end{array}$ & $\begin{array}{l}\text { The failure to align SME executive style } \\
\text { and strategy leads to performance loss. } \\
\text { Alignment is key when it comes to driving } \\
\text { change and innovation. }\end{array}$ & $\begin{array}{l}\text { Data gathered from } 241 \\
\text { companies on strategy and } \\
303 \text { companies on executive } \\
\text { style. }\end{array}$ \\
\hline 28 & $\begin{array}{l}\text { Mollahoseini \& } \\
\text { Ahmadkhani } \\
(2012) \\
\end{array}$ & $\begin{array}{l}\text { To identify strategy } \\
\text { obstacles. }\end{array}$ & $\begin{array}{l}\text { Number of obstacles such as } \\
\text { organizational, planning, managerial, staff, } \\
\text { and environmental obstacles identified. }\end{array}$ & $\begin{array}{l}\text { Study using interviews, focus } \\
\text { groups, and questionnaire, } \\
\text { with } 205 \text { respondents. }\end{array}$ \\
\hline 29 & $\begin{array}{l}\text { Al-Kandi et al. } \\
(2013)\end{array}$ & $\begin{array}{l}\text { To identify what } \\
\text { determines successful } \\
\text { strategy } \\
\text { implementation. }\end{array}$ & $\begin{array}{l}\text { Identification of process and personnel, } \\
\text { project, religion, and organizational factors. }\end{array}$ & $\begin{array}{l}\text { Study using questionnaire } \\
\text { with a sample of } 120 .\end{array}$ \\
\hline
\end{tabular}




\begin{tabular}{|c|c|c|c|c|}
\hline 30 & Pella et al. (2013) & $\begin{array}{l}\text { To study if and how } \\
\text { strategy } \\
\text { implementation } \\
\text { problems affect } \\
\text { strategy } \\
\text { implementation. }\end{array}$ & $\begin{array}{l}\text { Seven major obstacles identified. Included, } \\
\text { corporate scorecard, information } \\
\text { technology, performance appraisal, } \\
\text { management and financial support. }\end{array}$ & $\begin{array}{l}\text { A survey was conducted in } \\
60 \text { Indonesian companies, } \\
\text { and gathered } 194 \text { responses. }\end{array}$ \\
\hline 31 & Sabourin (2015) & $\begin{array}{l}\text { To understand the } \\
\text { underlying drivers of } \\
\text { performance in } \\
\text { strategy execution. }\end{array}$ & $\begin{array}{l}\text { Four drivers identified, rules, emotions, } \\
\text { initiatives, immediate action, and integrity. }\end{array}$ & $\begin{array}{l}\text { Study using survey } \\
\text { questionnaire to } 484 \\
\text { managers and } 180 \\
\text { responded. }\end{array}$ \\
\hline 32 & $\begin{array}{l}\text { Cebczynska } \\
\text { (2016) }\end{array}$ & $\begin{array}{l}\text { To verify to what extent } \\
\text { corporate strategy is } \\
\text { reflected in lower } \\
\text { managerial levels. }\end{array}$ & $\begin{array}{l}\text { Strategy implementation is most lacking on } \\
\text { an operational level and lack of training of } \\
\text { employees affects their awareness of the } \\
\text { corporate strategy. }\end{array}$ & $\begin{array}{l}\text { Research conducted in } 30 \\
\text { Polish enterprises by } \\
\text { questionnaire. }\end{array}$ \\
\hline 33 & $\begin{array}{l}\text { Köseoglu et al. } \\
\text { (2018) }\end{array}$ & $\begin{array}{l}\text { To examine potential } \\
\text { barriers to the } \\
\text { implementation of } \\
\text { strategic decisions. }\end{array}$ & $\begin{array}{l}\text { Identified } 41 \text { barriers to implementation } \\
\text { and at least } 20 \text { of them had influence on } \\
\text { performance and operation. }\end{array}$ & $\begin{array}{l}\text { Research conducted in } \\
\text { Turkish hotels by } \\
\text { questionnaire with } 118 \\
\text { responses. }\end{array}$ \\
\hline 34 & $\begin{array}{l}\text { Tawse et al. } \\
(2019)\end{array}$ & $\begin{array}{l}\text { To study top } \\
\text { managers' struggle } \\
\text { with the transition } \\
\text { between strategy } \\
\text { formulation and } \\
\text { implementation. }\end{array}$ & $\begin{array}{l}\text { Top managers tend to focus on strategy } \\
\text { formulation and planning but fail to } \\
\text { embrace the problem solving and } \\
\text { complexity of implementation. }\end{array}$ & $\begin{array}{l}\text { Research based on } \\
\text { interviews with CEOs. }\end{array}$ \\
\hline 35 & $\begin{array}{l}\text { Köseoglu et al. } \\
(2020)\end{array}$ & $\begin{array}{l}\text { To study how } \\
\text { managers perceive } \\
\text { strategic management } \\
\text { process. }\end{array}$ & $\begin{array}{l}\text { Managers in this study agree that } \\
\text { communication and people management } \\
\text { are the key success factors in strategy } \\
\text { implementation. }\end{array}$ & $\begin{array}{l}\text { Study using interviews with } \\
21 \text { managers. }\end{array}$ \\
\hline
\end{tabular}

Table 9 summarizes the research methods employed for all articles reviewed. The majority of studies gathered data using questionnaires, 12 studies used interviews, and 4 studies employed mixed methods of both interviews and questionnaires. Other studies employed focus groups (Mollahoseini \& Ahmadkhani, 2012), simulations (Walderseel \& Sheather, 1996), role play (Corboy \& O'Corrbui, 1999), observations (Okumus, 2001), panel discussions (Hrebiniak, 2006), and self-reporting real-life cases (Nutt, 1986). Some of the studies used one or more researched methods. In some cases the limitation of the research was not outlined in the reviewed articles. Nevertheless, identified limitations were related to the data collection methods, which in many cases were based on a single method, revealing a lack of triangulation (Cater \& Pucko, 2010; Govindarajan, 1988; Heide et al., 2002; Walderseel \& Sheather, 1996). Validity and generalization of some of the studies are limited based on the small sample used (Heide et al., 2002; Schaap, 2006). Some of the studies use selfreported data collection methods, which can be biased based on perspective (Cater \& Pucko, 2010; Håkonsson et al., 2012).
Table 9 Summary of research methods

\begin{tabular}{l|l}
\hline Research methods & Number \\
\hline Questionnaires & 22 \\
\hline Interviews & 13 \\
\hline Interviews \& questionnaires & 4 \\
\hline Case studies & 4 \\
\hline Multi-case study & 1 \\
\hline Focus groups & 1 \\
\hline Simulations & 1 \\
\hline Role play & 1 \\
\hline Observations & 1 \\
\hline Panel discussion & 1 \\
\hline Self-reported real-life cases & 1 \\
\hline & Source: The authors
\end{tabular}

\subsection{Analysis of obstacles and success factors}

Step three, as described in Section 2.4, focuses on the analysis of the content of the papers. First, Table 10 shows the 16 obstacles and the 18 success factors identified in the 35 articles and demonstrates the range of factors identified from the literature sources. The factors have been grouped based on the 5Ps model introduced by Pryor et al. (2007) into purpose, principles, process, people, and performance. Second, Table 10 illustrates a pattern that emerged when comparing the content of all the obstacles and the 
success factors: Many of the obstacles and the success factors identified can simultaneously be viewed as obstacles hindering implementation and factors necessary for successful implementation.
Based on this analysis related factors affecting strategy implementation are paired (see the grey area and the arrow in Table 10) and the articles that reference each factor are reported in parentheses.

Table 10 Related obstacles and success factors influencing strategy implementation, grouped according to the 5Ps Model Pryor et al. (2007). (Article numbers in Table $5-8$ are given in parentheses)

\begin{tabular}{|c|c|c|}
\hline Obstacles & & Success factors \\
\hline \multicolumn{3}{|c|}{ Purpose } \\
\hline Inadequate leadership $(5,14,19,21,24)$ & $<-->>$ & $\begin{array}{l}\text { Leadership styles and motivation } \\
(11,22,23,25,27,29)\end{array}$ \\
\hline Goals and tasks not defined $(5,12,13,19,21,28,30)$ & $<--->$ & Goals and objectives linked to strategy $(1,18,31,34)$ \\
\hline $\begin{array}{l}\text { Management style and lack of support } \\
(4,7,13,14,24,28)\end{array}$ & $<--->$ & $\begin{array}{l}\text { Management style and support effects } \\
\text { implementation (3) }\end{array}$ \\
\hline Unclear strategy $(14,21,24,33)$ & $<-->>$ & Well-defined strategy $(2,6,35)$ \\
\hline \multirow[t]{3}{*}{ External barriers $(5,17,28)$} & & Tactics support implementation (6) \\
\hline & & Broad strategy formulation (9) \\
\hline & & Different strategies for business units (8) \\
\hline \multicolumn{3}{|c|}{ Principles } \\
\hline Inadequate change management $(4,21,24,33)$ & $<-->>$ & Change management lead by executives $(23,25,29)$ \\
\hline Cultural problems $(17,21,24,28)$ & $<-->$ & Shaping corporate culture (22) \\
\hline No guidelines to implement $(21,32)$ & & Organizational learning $(15,22)$ \\
\hline \multicolumn{3}{|c|}{ Process } \\
\hline Poor communication $(14,17,24)$ & $<-->$ & Frequent communication $(16,18,20,22,26,29,35)$ \\
\hline Lack of time $(4,5,12)$ & $<-->>$ & Timeframes and priorities set $(18,20,29,34,35)$ \\
\hline Lack of resources $(17,24)$ & $<-->>$ & Existing resources $(22,25,35)$ \\
\hline \multicolumn{3}{|l|}{ Unexpected problems $(5,12,33)$} \\
\hline \multicolumn{3}{|c|}{ People } \\
\hline Limited commitment and understanding $(13,19,28)$ & $<-->$ & Shared understanding $(2,9,10,16,18,20,31,35)$ \\
\hline \multicolumn{3}{|l|}{ Inadequate staff capabilities $(5,14,17,19,24,32)$} \\
\hline \multicolumn{3}{|c|}{ Performance } \\
\hline Limited alignment and coordination $(5,12,14)$ & $<-->>$ & Alignment and integration $(1,15,18,22,27,31)$ \\
\hline \multirow[t]{3}{*}{$\begin{array}{l}\text { IT do not support or monitor strategy } \\
(4,5,12,19,24,33)\end{array}$} & & Monitoring strategy $(22,35)$ \\
\hline & & Staff accountable for task and action $(20,23,26,31)$ \\
\hline & & Tying awards with implementation $(18,20,22)$ \\
\hline
\end{tabular}

Figure 3 shows the obstacles and the success factors affecting strategy implementation grouped graphically based on the five elements of purpose, principles, process, people, and performance from the 5Ps model (Pryor et al., 2007). These five elements in Figure 3 are displayed in one circle each. Inside each circle both success factors and obstacles are displayed in smaller ovals. The grey ovals in Figure 3 connect identified obstacles within each element and the success factors which can simultaneously be viewed as obstacles hindering implementation and factors necessary for successful implementation. These factors are all demonstrated in grey and are called dualistic factors hereafter. Outside the grey ovals are obstacles in each element and inside are the success 
factors in each element, all demonstrated in white.

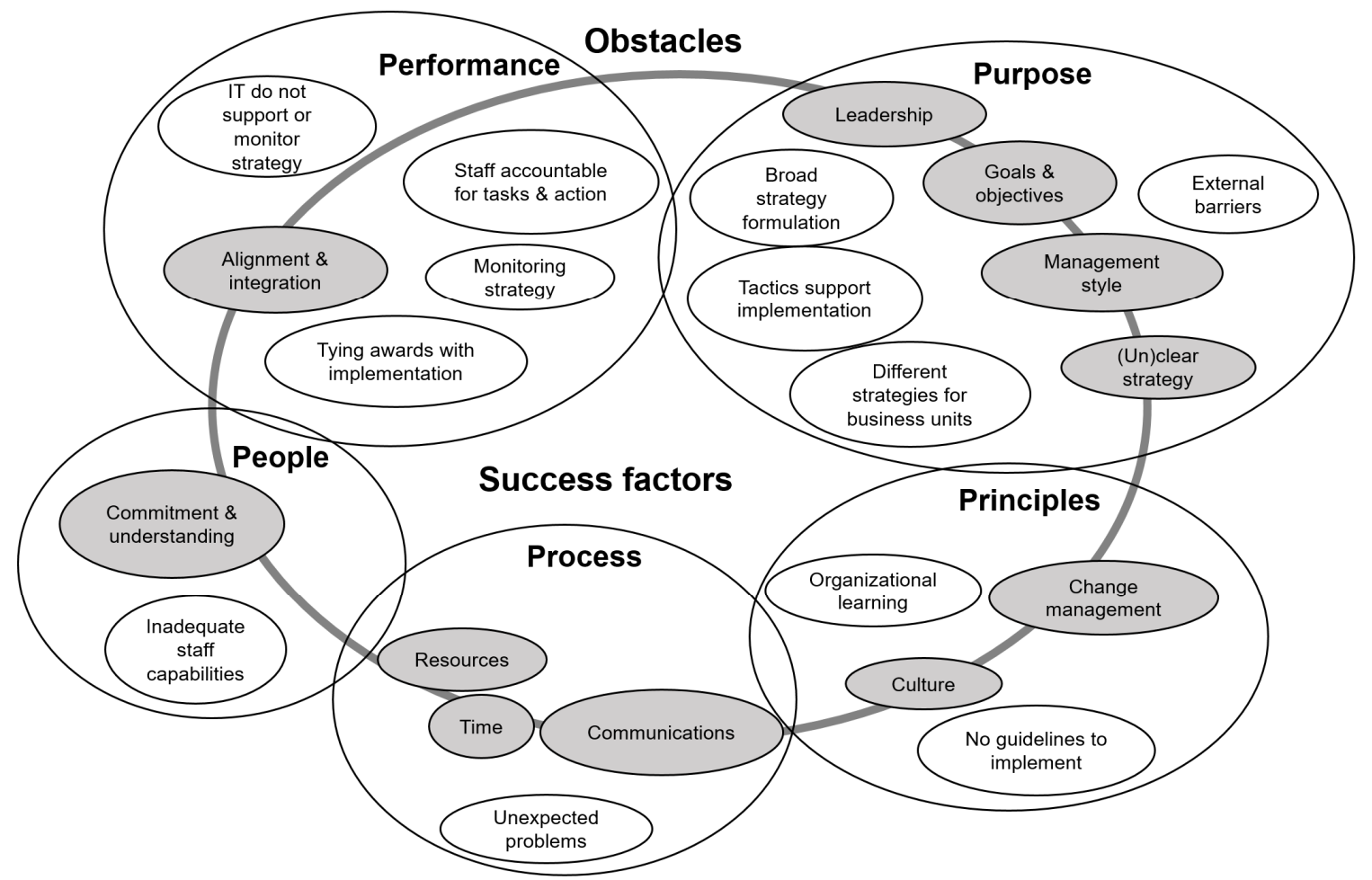

Figure 3 Strategy implementation obstacles and success factors Source: The authors

The following discussion centres on the dualistic factors identified in Table 10 presented in Figure 3. The first of the dualistic factors in the purpose element is leadership, which focuses on leadership support, motivation, and style influencing implementation. Shah (2005) underlined that commitment and support refers to involvement and overall responsibility of top management, which should be accountable for the implementation process. This responsibility, according to Shah, cannot lie anywhere else in the organization, as top managers are accountable for the performance of an organization as a whole. The second dualistic factor brings forth the importance of defining objectives, setting goals, and linking them to the business strategy and the competitive environment as vital aspects related to implementation (Beer \& Eisenstat, 2000). Setting key performance indicators is not only inadequate in many organizations but is also seen as challenging, and this is even harder to accomplish at lower levels in organizations (Aaltonen \& Ikävalko, 2002). The lack of indicators, objectives and goal setting can, therefore, hinder implementation. Employees often do not have a road map for implementation, as there are no clear key indicators for the performance of each person or position (Pella et al., 2013). The third dualistic factor is management style, which has been identified in a number of studies. Wernham emphasized that top management support was important for successful implementation and is one of the rare points on which most experts can agree (1985). Beer and Eisenstat (2000) indicated that frequent absence of senior management during strategic decision-making would affect the implementation. Some studies have also shown that senior management tends to withdraw when implementation begins and their lack of support can consequently affect the implementation (Corboy \& O'Corrbui, 1999). The fourth dualistic factor highlights the importance of having a clear strategy for successful implementation. A number of researchers have come to this conclusion (Alamsjah, 2011; Beer \& Eisenstat, 2000; Cater \& Pucko, 2010; Köseoglu et al., 2018). Hrebiniak (2006) stressed that poor or vague strategy limits the implementation effort and therefore is a major obstacle as good execution cannot overcome various shortcomings of a bad strategy or poor 
implementation efforts.

There are two dualistic factors in the principle element, change management and culture. Change management was identified by Hrebiniak (2006) and he determined that the inability to manage change and the importance of reducing resistance to change was the topmost challenge and obstacle for managers. Cater and Pucko (2010) found that managers lack capabilities to implement change management in Slovenian companies, which supports Hrebiniak's conclusion. Crittenden and Crittenden (2008) found that company internal culture plays a vital role as a driving force in successful implementation. They also found that different cultures between silos can have a negative influence on implementation (Crittenden \& Crittenden, 2008). The importance of culture has been identified as a major obstacle or success factor by a number of authors (Brenes, 2008; Heide, 2002; Köseoglu et al., 2018; Sorooshian et al., 2010).

The first dualistic factor in the process element is communications, which highlights the importance of frequent and clear communication and the issues which can arise with a lack of communication. Alamsjah (2011) found that it is not enough just to have a clear strategy it needs to be communicated through the organization and be translated into execution plans. Rapert et al. (2002) highlighted that vertical communication and shared understanding play a key role in the process. Similar findings are discussed by Schaap (2006) on the importance of communications to help employees understand the strategy being implemented. The second dualistic factor in the process element is time and importance of timeframes being set. A number of studies have shown the importance of realistic timeframes, as strategy implementation takes more time than commonly allocated (Al-Ghamdi, 1988; Alexander, 1985). Al-Kandi et al. (2013) indicated that, if timeframes were set, they had to be followed up with detailed planning and implementation must be put ahead of other managerial commitments. The resource factor is the third factor in the process element. Crittenden and Crittenden (2008) found it was not only important for companies to have access to resources but also to decide when and where to allocate them, whether it is money, people, time, or capabilities.

The only factor in the people element emphasizes that commitment and shared understanding of the strategy influences successful implementation (Rapert et al., 2002), but so does the importance of building up consensus in the implementation process to foster shared attitudes and values (Schaap, 2006).

Last, performance element has one dualistic factor, which is alignment and coordination which has been identified by a number of scholars. Strategy alignment is the important process of linking the organization's structure and resources with its strategy and external business environment (Aaltonen \& Ikävalko, 2002; Al-Ghamdi, 1998; Beer \& Eisenstat, 2000; Håkonsson et al., 2012; Okumus, 2001).

\section{Conclusion}

Research on strategy implementation is still in its early days, with a limited number of empirical studies completed. Most of the effort has historically been focused on strategy formulation and process and less on strategy implementation, let alone studies grounded in empirical data.

The findings suggest and synthesize that empirical research has so far identified 16 obstacles that can hinder strategy implementation and 18 key success factors that can facilitate implementation. This study also draws the attention to, and highlights, factors that are dualistic, meaning that they contain both elements of obstacles and key success factors. The dualistic factors, or elements, should therefore be considered in the strategy implementation process.

The theoretical implication of the study is that it provides an overview of major empirical research concerning strategy implementation obstacles and success factors conducted from 1980 until 2020. Older studies providing the review of the literature reach back to 2010. Furthermore, previous literature reviews combine a mixture of conceptual and empirical research, as presented in Table 1, and have left out number of empirical papers that are included in this particular review. Therefore, this systematic review offers a more holistic overview of implementation obstacles, success factors, and dualistic factors, than previous studies.

The paper also enriches the practical knowledge on implementation obstacles, success factors, and dualistic factors which are of relevance for those implementing strategies, such as managers and other implementers. Another benefit offered to practitioners is how these factors are categorized based on the five critical elements presented in the 5Ps model including the concepts of strategy implementation, namely: purpose, principles, 
processes, people, and performance.

Ample room exists for further study on strategy implementation obstacles and understanding how to overcome them, a problem that seems to occur repeatedly in the strategic process. Studies that combine the identification of obstacles and uncover workable solutions are lacking. Additionally, a substantial lack of studies exists on employee influence on strategy implementation, both positive and negative aspects, which may be crucial to gain a better understanding of why and how so many strategy implementation projects fail, or succeed in some cases. Strategy implementation literature could benefit from a greater number of studies employing observation, focus groups, and panel discussions, which could add value and new perspectives to the understanding of the topic in question. The differences among industries, countries, or cultures regarding implementation have not yet been explored at any depth.

One of the limitations to this study is that selection bias of authors and subjectivity may have influenced the exclusion of articles but several techniques were used to mitigate this risk. This review relies on databases that support keyword search, where search strings must be identifiable. This approach used could overlook important literature and restrict accessibility of sources. Furthermore, this review article focuses solely on full-text articles published in English and last, a small number of articles analysed limits the generalizability of the findings.sm

\section{References}

Aaltonen, P., \& Ikävalko, H. (2002). Implementing strategies successfully. Integrated Manufacturing Systems, 13(6), 415-418.

https://doi.org/10.1108/09576060210436669

Alamsjah, F. (2011). Key success factors in implementing strategy: Middle-level managers' perspectives. Procedia - Social and Behavioral Sciences, 24, 1444-1450. https://doi.org/10.1016/j.sbspro.2011.09.049

Alexander, L. D. (1985). Successfully implementing strategic decisions. Long Range Planning, 18(3), 9197.

https://doi.org/10.1016/0024-6301(85)90161-X

Al-Ghamdi, S. M. (1998). Obstacles to successful implementation of strategic decisions: The British experience. European Business Review, 98(6), 322327.

http://dx.doi.org/10.1108/09555349810241590

Alharthy, A. H., Khan, F., Rashid, H., \& Pagliari, R. (2017). Identification of Strategy Implementation Influencing Factors and Their Effects on the Performance: International Journal of Business and Social Science, 8(1), 34-44. http://dspace.lib.cranfield.ac.uk/handle/1826/11802

Al-Kandi, I., Asutay, M., \& Dixon, R. (2013). Factors influencing the strategy implementation process and its outcomes: Evidence from Saudi Arabian banks. Journal of Global Strategic Management, 2(7), 5-15. https://doi.org/10.20460/JGSM.2013715662

Allio, M. K. (2005). A short, practical guide to implementing strategy. Journal of Business Strategy, 26(4), 12-21. https://doi.org/10.1108/02756660510608512

Atkinson, H. (2006). Strategy implementation: A role for the balanced scorecard? Management Decision, 44(10), 1441-1460.

https://doi.org/10.1108/00251740610715740

Baumgartner, R. J. (2014). Managing corporate sustainability and CSR: A conceptual framework combining values, strategies and instruments contributing to sustainable development. Corporate Social Responsibility and Environmental Management, 21(5), 258-271.

https://doi.org/10.1002/csr.1336

Beer, M., \& Eisenstat, R. A. (2000). The silent killers of strategy implementation and learning. Sloan Management Review, 41(4), 29-40.

Blahová, M., \& Knápková, A. (2010). Effective strategic action: From formulation to implementation. 2010 International Conference of Economics, Business and Management, 61-65. Manila, Philippines.

Bourgeois, L. J. (1980). Performance and consensus: Summary. Strategic Management Journal, 1(3), 227-248.

https://doi.org/10.1002/smj.4250010304

Brenes, E. R., Mena, M., \& Molina, G. E. (2008). Key success factors for strategy implementation in Latin America. Journal of Business Research, 61(6), 590598.

http://dx.doi.org/10.1016/j.jbusres.2007.06.033

Cândido, C. J. F., \& Santos, S. P. (2015). Strategy implementation: What is the failure rate? Journal of Management \& Organization, 21(2), 237-262. https://doi.org/10.1017/jmo.2014.77

Cater, T., \& Pucko, D. (2010). Factors of effective strategy implementation: Empirical evidence from Slovenian business practice. Journal for East European Management Studies, 15(3), 207-236. https://doi.org/10.5771/0949-6181-2010-3-207

Corboy, M., \& O'Corrbui, D. (1999). The seven deadly sins of strategy. Management Accounting: Magazine for Chartered Management Accountants, 77(10), 29-30.

Crittenden, V. L., \& Crittenden, W. F. (2008). Building a capable organization: The eight levers of strategy implementation. Business Horizons, 51(4), 301-309. https://doi.org/10.1016/j.bushor.2008.02.003

Daft, R. L., \& Macintosh, N. B. (1984). The nature and use of formal control systems for management control and strategy implementation. Journal of Management, 10(1), 43-66. https://doi.org/10.1177/014920638401000105

David, F. R., \& David, F. R. (2013). Strategic management: Concepts and cases: A competitive advantage approach. Upper Saddle River, NJ: Pearson.

De, F. J. A., \& Janssen, A. (2001). Implementing a strategy successfully. Measuring Business Excellence, 5(4), 46. https://doi.org/10.1108/13683040110411223

Engert, S., \& Baumgartner, R. J. (2016). Corporate sustainability strategy - bridging the gap between formulation and implementation. Journal of Cleaner Production, 113, 822-834. 
https://doi.org/10.1016/j.jclepro.2015.11.094

Floyd, S. W., \& Wooldridge, B. (1997). Middle management's strategic influence and organizational performance. Journal of Management Studies, 34(3), 465-485. https://doi.org/10.1111/1467-6486.00059

Gębczyńska, A. (2016). Strategy implementation efficiency on the process level. Business Process Management Journal, 22(6), 1079-1098. https://doi.org/10.1108/BPMJ-01-2016-0004

Galbraith, J. R., \& Nathanson, D. A. (1978). Strategy implementation: The role of structure and process (7th ed.). St Paul, MN: West Publications.

Gębczyńska, A. (2016). Strategy implementation efficiency on the process level. Business Process Management Journal, 22(6), 1079-1098. https://doi.org/10.1108/BPMJ-01-2016-0004

Giles, W. D. (1991). Making strategy work. Long Range Planning, 24(5), 75-91. https://doi.org/10.1016/0024-6301(91)90254-L

Govindarajan, V. (1988). A contingency approach to strategy implementation at the business-unit level: Integrating administrative mechanisms with strategy. Academy of Management Journal, 31(4), 828-853. https://doi.org/10.5465/256341

Gupta, A. K., \& Govindarajan, V. (1984). Business unit strategy, managerial characteristics, and business unit effectiveness at strategy implementation. Academy of Management Journal, 27(1), 25-41. https://doi.org/10.2307/255955

Guth, W. D., \& Macmillan, I. C. (1986). Strategy implementation versus middle management selfinterest. Strategic Management Journal, 7(4), 313-327. https://doi.org/10.1002/smj.4250070403

Håkonsson, D. D., Burton, R. M., Obel, B., \& Lauridsen, J. T. (2012). Strategy implementation requires the right executive style: Evidence from Danish SMEs. Long Range Planning, 45(2-3), 182-208. https://doi.org/10.1016/j.Irp.2012.02.004

Hambrick, D. C., \& Cannella, A. A. (1989). Strategy implementation as substance and selling. Academy of Management Executive, 3(4), 278-285. https://doi.org/10.5465/AME.1989.4277401

Heide, M., Grønhaug, K., \& Johannessen, S. (2002). Exploring barriers to the successful implementation of a formulated strategy. Scandinavian Journal of Management, 18(2), 217-231. https://doi.org/10.1016/S0956-5221(01)00007-0

Hrebiniak, L. G. (2006). Obstacles to effective strategy implementation. Organizational Dynamics, 35(1), 12 31. https://doi.org/10.1016/j.orgdyn.2005.12.001

Hrebiniak, L. G. (2013). Making strategy work: Leading effective execution and change. Upper Saddle River, $\mathrm{NJ}$ : Pearson Education.

Hrebiniak, L. G., \& Joyce, W. F. (1984). Implementing strategy. New York, NY: Macmillan.

Hrebiniak, L. G., \& Snow, C. C. (1982). Top-management agreement and organizational performance. Human Relations, 35(12), 1139-1157. https://doi.org/10.1177/001872678203501205

Kaplan, R. S., \& Norton, D. P. (2000). Having trouble with your strategy? Then map it. Harvard Business Review, 78(5), 167-176.
Kaplan, R. S., \& Norton, D. P. (2001). The strategy-focused organization. Strategy \& Leadership, 29(3), 41-42. https://doi.org/10.1108/sl.2001.26129cab.002

Kaplan, R. S., \& Norton, D. P. (2004). Measuring the strategic readiness of intangible assets. Harvard Business Review, 82(2), 52-63.

Kaplan, R. S., \& Norton, D. P. (2008). Mastering the management system. Harvard Business Review, 86(1), 29-48.

Klettner, A., Clarke, T., \& Boersma, M. (2014). The governance of corporate sustainability: empirical insights into the development, leadership and implementation of responsible business strategy. Journal of Business Ethics, 122(1), 145-165. https://doi.org/10.1007/s10551-013-1750-y

Köseoglu, M. A., Yazici, S., \& Okumus, F. (2018). Barriers to the implementation of strategic decisions: evidence from hotels in a developing country. Journal of Hospitality Marketing \& Management, 27(5), 514-543. https://doi.org/10.1080/19368623.2018.1402723

Köseoglu, M. A., Altin, M., Chan, E.,\& Aldrag, A. F., (2020). What are the key success factors for strategy formulation and implementation? Perspective of managers in hotel industry. International Journal of Hospitality Management, 89(6), 1-9. https://doi.org/10.1016/j.ijhm.2020.102574

Li, Y., Guo-hui, S., \& Eppler, M. J. (2010). Making strategy work: a literature review on the factors influencing strategy implementation. In F. W. Kellermanns \& M. Mazzola (Eds.), Handbook of research on strategy process (pp. 165-183). Cheltenham, England: Edward Elgar.

Littler, K., Aisthorpe, P., Hudson, R., \& Keasey, K. (2000). A new approach to linking strategy formulation and strategy implementation: an example from the UK banking sector. International Journal of Information Management, 20(6), 411-428. https://doi.org/10.1016/S0268-4012(00)00036-0

Miller, S. (1997). Implementing strategic decisions: four key success factors. Organization Studies, 18(4), 577-602. https://doi.org/10.1177/017084069701800402

Miller, S., \& Friesen, P. H. (1978). Archetypes of strategy formulation. Management Science, 24(9), 921-933. https://doi.org/10.1287/mnsc.24.9.921

Mintzberg, H., \& Lampel, J. (1999). Reflecting on the strategy process. Sloan Management Review, 40(3), 21-30.

Mintzberg, H., \& McHugh, A. (1985). Strategy formation in an adhocracy. Administrative Science Quarterly, 30(2), 160-197. https://doi.org/10.2307/2393104

Mollahoseini, A., \& Ahmadkhani, H. (2012). Surveying and identifying the factors affecting successful implementation of business strategies in companies of Fars Province industrial towns (case study: companies of food industries). International Journal of Business and Social Science, 3(1), 265-272.

Nakano, D., \& Muniz Jr., J. (2018). Writing the literature review for empirical papers. Production, 28, e2070086. https://doi.org/10.1590/0103-6513.20170086

Noble, C. H. (1999a). The eclectic roots of strategy implementation research. Journal of Business Research, 45(2), 119-134. https://doi.org/10.1016/S0148-2963(97)00231-2 
Noble, C. H. (1999b, November 1). Building the strategy implementation network. Retrieved September 27, 2018, from http://link.galegroup.com/apps/doc/A58381515/AONE?s id=googlescholar

Nutt, P. C. (1983). Implementation approaches for project planning. Academy of Management Review, 8(4), 600611. https://doi.org/10.5465/amr.1983.4284660

Nutt, P. C. (1986). Tactics of implementation. Academy of Management Journal, 29(2), 230-261. https://doi.org/10.5465/256187

Okumus, F. (2001). Towards a strategy implementation framework. International Journal of Contemporary Hospitality Management, 13(7), 327-338. https://doi.org/10.1108/09596110110403712

Okumus, F. (2003). A framework to implement strategies in organizations. Management Decision, 41(9), 871-882. https://doi.org/10.1108/00251740310499555

Olson, E. M., Slater, S. F., \& Hult, G. T. M. (2005). The importance of structure and process to strategy implementation. Business Horizons, 48(1), 47-54. https://doi.org/10.1016/j.bushor.2004.10.002

Pella, M. D. A., Sumarwan, U., Daryanto, A., \& Kirbrandoko, K. (2013). Factors affecting poor strategy implementation. Gadjah Mada International Journal of Business, 15(2), 183-204. https://doi.org/10.22146/gamaijb.5702

Pettigrew, A. M. (1992). The character and significance of strategy process research. Strategic Management Journal, 13(S2), 5-16. https://doi.org/10.1002/smj.4250130903

Pryor, M. G., Anderson, D., Toombs, L. A., \& Humphreys, J. H. (2007). Strategic implementation as a core competency. Journal of Management Research, 7(1) 3-17.

Rapert, M. I., Velliquette, A., \& Garretson, J. A. (2002). The strategic implementation process: Evoking strategic consensus through communication. Journal of Business Research, 55(4), 301-310. https://doi.org/10.1016/S0148-2963(00)00157-0

Sabourin, V. (2015). Strategy execution: Five drivers of performance. Journal of Strategy and Management; Bingley, 8(2), 127-138. https://doi.org/10.1108/JSMA-06-2014-0048

Safdary Ranjbar, M., Akbarpour Shirazi, M., Lashkar Blooki., M. (2014). Interation among intra-organizational factors effective in successful strategy execution: an analytical view. Journal of Strategy and Management, 7(2), 127-154. https://doi.org/10.1108/JSMA-05-2013-0032

Schaap, J. (2006). Toward strategy implementation success: an empirical study of the role of senior-level leaders in the Nevada gaming industry. UNLV Gaming Research \& Review Journal, 10(2), 13-37.
Shah, A. M. (2005). The foundations of successful strategy implementation: overcoming the obstacles. Global Business Review, 6(2), 293-302. https://doi.org/10.1177/097215090500600208

Simons, R. (1994). How new top managers use control systems as levers of strategic renewal. Strategic Management Journal, 15(3), 169-189. https://doi.org/10.1002/smj.4250150301

Sorooshian, S., Norzima, Z., Yusof, I., \& Rosnah, Y. (2010). Effect analysis on strategy implementation drivers. World Applied Sciences Journal, 11(10), 1255-1261.

Tawse, A., Tabesh, P. (2020). Strategy implementation: a review and an introductory framework. European Management Journal. 39(1), 22-23. https://doi.org/10.1016/j.emj.2020.09.005

Tawse, A., Patrick, V. M., \& Vera, D. (2019). Crossing the chasm: Leadership nudges to help transition from strategy formulation updates to strategy implementation. Business Horizons, 62(2), 249-257. https://doi.org/10.1016/j.bushor.2018.09.005

Torraco, R. J. (2016). Writing integrative literature reviews: Using the past and present to explore the future. Human Resource Development Review, 15(4), 404428.

https://doi.org/10.1177/1534484316671606

Walderseel, R., \& Sheather, S. (1996). The Effects of Strategy Type on Strategy Implementation Actions. Human Relations, 49(1), 105-122. https://doi.org/10.1177/001872679604900105

Waterman, R. H., Peters, T. J., \& Phillips, J. R. (1980). Structure is not organization. Business Horizons, 23(3), 14-26.

Webster, J., \& Watson, R. T. (2002). Analyzing the past to prepare for the future: Writing a literature review. MIS Quarterly, 26(2), XIII-XXIII.

Wernham, R. (1985). Obstacles to strategy implementation in a nationalized industry. Journal of Management Studies, 22(6), 632-648. https://doi.org/10.1111/j.1467-6486.1985.tb00015.x

Wohlin, C. (2014). Guidelines for snowballing in systematic literature studies and a replication in software engineering. EASE '14: 18th International Conference on Evaluation and Assessment in Software Engineering. 38, 1-10. https://doi.org/10.1145/2601248.2601268

Wooldridge, B., \& Floyd, S. W. (1990). The strategy process, middle management involvement, and organizational performance. Strategic Management Journal, 11(3), 231-241. https://doi.org/10.1002/smj.4250110305
Schilit, W. K. (1987). An examination of the influence of middle-level managers in formulating and implementing strategic decisions. Journal of Management Studies, 24(3), 271-293. https://doi.org/10.1111/j.1467-6486.1987.tb00703.x

$\bowtie$ Correspondence 
Vigfússon et al. Obstacles to strategy implementation and success factors: a review of empirical literature

\section{Kristján Vigfússon}

Reykjavík University, School of Social Science

Menntavegi 1, 103 Reykjavík, Iceland

$\mathrm{Ph}:+3548602077$

E-mail: kristjanv@ru.is 